\title{
Characterization and comparison of gut microbiomes in nine species of parrots in captivity
}

\author{
Hongyi Liu ${ }^{1} \cdot$ Zhiwei Chen ${ }^{1} \cdot$ Ge Gao ${ }^{1} \cdot$ Chenghe Sun ${ }^{1} \cdot$ Yudong $\mathrm{Li}^{2} \cdot$ Ying Zhu $^{2}$ (D)
}

Received: 1 November 2018 / Accepted: 4 March 2019 / Published online: 25 March 2019

(C) The Author(s) 2019

\begin{abstract}
Gut microbiomes have profound effects on the health of hosts. About $28 \%$ of extant parrot species are threatened with extinction. To inform conservation efforts, we characterized and compared the gut microbiomes in nine species of parrots in captivity. The core gut microbiome of parrots was dominated by three bacterial phyla: the Firmicutes, Proteobacteria, and Actinobacteria. This core gut microbiome is similar to that in other herbivorous birds. There were no statistical differences in the diversity of gut microbiomes among parrot species, but significant variations in richness were detected. Individuals of the same species had more similar gut microbial composition to each other than to other species, indicating the potential role of host ancestry on shaping gut microbiomes. However, gut microbiomes did not cluster according to host species. Furthermore, Lactobacillus might influence the gut microbial composition of parrots. These results can contribute to improving our understanding of the basic characteristics of parrot gut microbiomes and help with their conservation.
\end{abstract}

Keywords Captivity $\cdot$ Ex-situ conservation $\cdot$ Gut microbiome $\cdot$ Host phylogeny $\cdot$ Lactobacillus $\cdot$ Parrot species

\section{Introduction}

Bird diversity is an important component of biodiversity, which plays an important role in ecological balance (Sekercioglu 2006). Bird populations have been declining at the global scale due to human induced pressure (Simberloff 2001; Xu et al. 2016). According to BirdLife data in the 2018 IUCN red list, $13 \%$ of the known bird species are threatened (IUCN 2018). Many species of the order Psittaciformes (parrots) are threatened with extinction (Berg and Bennett 2010; Olah et al. 2016; Heinsohn et al. 2018).

Parrots are divided into three families (Psittacidae, Cacatuidae, Strigopidae), and are widely distributed in the tropical and subtropical habitats of 124 countries between latitudes 35 degrees north and 56 degrees south (Olah et al. 2016). Habitat loss and fragmentation driven by agriculture and urbanization

Ying Zhu

so_zy2003@126.com

1 Co-Innovation Center for Sustainable Forestry in Southern China, College of Biology and the Environment, Nanjing Forestry University, Nanjing 210037, China

2 Sichuan Province Laboratory for Natural Resources Protection and Sustainable Utilization, Sichuan Provincial Academy of Natural Resource Sciences, Chengdu 610015, China have reduced the population sizes of parrots (Olah et al. 2016; Heinsohn et al. 2018). In addition, parrots are popular as pets due to their colorful plumage and capacity to mimic human speech (Berg and Bennett 2010; Bradbury and Balsby 2016), and they are the most common avians in the wildlife trade (Bush et al. 2014). With the confluence of these negative factors, about $28 \%$ of extant parrot species are critically endangered, endangered, or vulnerable (IUCN 2018). Fortunately, many parrot species have been placed in zoos due to their ornamental value and survival predicament (Rodríguez-López 2016). Understanding the common physiological condition of different parrot species in captivity is particularly important for their conservation.

Gut microbiomes are extremely important to the health of hosts (Andoh 2016; Wang et al. 2016). Gut microbiomes are involved in many key physiological and biochemical functions of hosts, such as nutrition metabolism, vitamin synthesis, and the maturation of the gut immune system (Andoh 2016; Wang et al. 2016). Gut microbial communities consist of stable and transient inhabitants that can assemble through purely stochastic processes associated with the environment or by interactions with hosts. If host-microbiome interactions impact assembly patterns, there can be concordance between host evolutionary histories and the ecological similarities of microbial community structures (Brooks et al. 2017; Kohl et al. 2018). Similarities in gut microbiomes among species 
are usually associated with host phylogenies (Sanders et al. 2014; Brooks et al. 2017; Kohl et al. 2018). Besides host phylogenies, several factors such as diet and health condition play important roles in shaping gut microbiomes (Rothschild et al. 2018). The gut microbiome of individuals in the same species and even in the same individual may vary dramatically with diet and health condition (Nelson et al. 2013; Waite et al. 2014; Kers et al. 2018).

The basic characteristics and dynamic change in the gut microbiomes of threatened birds have drawn increasing attention (Waite et al. 2012; Waite et al. 2014; Yang et al. 2016; Zhao et al. 2017). However, to our knowledge, there are few studies investigating the gut microbiomes of parrots and their dynamic changes, except the kakapo (Strigops habroptila) (Waite et al. 2012; Waite et al. 2014). The premise of understanding the potentially important roles of gut microbiomes in parrot physiological and biochemical functions is that the information on gut microbial communities of the parrots is collected and recorded systematically (Waite et al. 2012).

In this study, we used 16S rRNA high-throughput sequencing to investigate the microbial communities of nine species of parrots (seven species of Psittacidae and two species of Cacatuinae) in captivity. Then, we characterized core and unique gut microbiomes by comparing the different parrot species. This study contributes to our understanding of the basic characteristics of the gut microbiomes in parrots in captivity, and thereby promotes ex situ conservation of threatened parrot species.

\section{Materials and methods}

\subsection{Subjects and sampling}

One fecal sample was collected from 37 individuals from nine species of parrots. The parrot species belonged to eight genera in two families, including Psittacus erithacus $(n=4)$, Ara ararauna $(\mathrm{n}=4)$, Amazona aestiva $(n=5)$, Myiopsitta monachus $(\mathrm{n}=5)$, Psittacula eupatria $(n=3)$, Psittacula derbiana $(\mathrm{n}=5)$, Eclectus roratus $(\mathrm{n}=5)$, Cacatua ducorpsii $(\mathrm{n}=3)$ and Probosciger aterrimus $(\mathrm{n}=3)$. Conspecifics were raised together in cages in the Nanjing Hongshan Forest Zoo. The cages were arranged side-by-side. Little is known about sex, age, and relatedness of these parrots, but they were all healthy adults based on descriptions from the zookeeper and veterinarian. They were fed the same diet daily, which consisted of abundant commercial feed (Mazuri, USA) (which contained Lactobacillus), fruits, and vegetables. During sampling, the parrots ate in their regular cages, were then moved to individual cages, and samples were collected one to two hours after feeding. To ensure accuracy, cages were cleaned prior to sampling. The liquid components of the fecal samples were abandoned, and the solid components, specifically the interiors, were collected to prevent contamination by environmental microorganisms. All 37 fecal samples were collected immediately after excretion and stored in liquid nitrogen for laboratory experiments.

\subsection{DNA extraction, PCR amplification, and sequencing}

Total DNA from fecal samples was extracted using the hexadecyltrimethylammonium bromide method. DNA templates were dissolved in $40 \mu \mathrm{L}$ water. The concentrations and purities of the DNA extractions were measured using a Qubit ${ }^{\circledR}$ 2.0 Fluorometer (Thermo Fisher Scientific, USA) and $1 \%$ agarose gel electrophoresis. Then, DNA templates with concentrations between 29.60 to $47.20 \mathrm{ng} / \mu \mathrm{L}$ were used for PCR. The V3-V4 region of the 16S rRNA gene was amplified using the primers $341 \mathrm{~F}$ (5'-CCTAYGGGRBGCASCAG-3') and 806R (5'-GGACTACNNGGGTATCTAAT-3'). To ensure accuracy of the amplicons, all PCR reactions were carried out with Phusion ${ }^{\circledR}$ High-Fidelity PCR Master Mix (New England Biolabs, USA). Reaction conditions were $98{ }^{\circ} \mathrm{C}$ for $1 \mathrm{~min}$, followed by 30 cycles of $98{ }^{\circ} \mathrm{C}$ for $10 \mathrm{~s}, 50{ }^{\circ} \mathrm{C}$ for $30 \mathrm{~s}$, and $72{ }^{\circ} \mathrm{C}$ for $30 \mathrm{~s}$. PCR products were mixed in equidensity ratios. Mixed PCR products ranging from 400 to $450 \mathrm{bp}$ were recovered from the $2 \%$ agarose gel using a GeneJET Gel Extraction Kit (Thermo Fisher Scientific, USA). The libraries used for sequencing were generated using an Ion Plus Fragment Library Kit 48 rxns (Thermo Fisher Scientific, USA). The quality of the libraries was determined using a Qubit ${ }^{\circledR}$ 2.0 Fluorometer. The high-quality libraries were sequenced on an Ion S5 ${ }^{\mathrm{TM}}$ XL platform (Thermo Fisher Scientific, USA).

\subsection{Data analyses}

Single-end reads were assigned to samples based on unique barcodes using Cutadapt v1.9.1 (Martin 2011). Quality filtering of the reads was performed according to the Cutadapt v1.9.1 quality controlled process (Martin 2011). By comparing reads with the reference database, chimeric sequences were detected and removed using the UCHIME algorithm (Edgar et al. 2011; Haas et al. 2011). After deleting invalid sequences, clean sequences were clustered into operational taxonomic units (OTUs) at a 97\% identity level using Uparse v7.0.1001 (Edgar 2013). A representative sequence for each OTU was screened for further annotation. Annotation of each representative sequence was performed in Mothur v1.35.1 based on the SILVA rRNA gene database (Schloss et al. 2009; Quast et al. 2013). OTU abundance information was normalized using a standard sequence number corresponding to the 
Table 1 The main quality control parameters of the 16S rRNA gene high-throughput sequencing

\begin{tabular}{lllll}
\hline Parameters & Minimum & Maximum & Average \pm SD & Total \\
\hline Raw reads & 69,847 & 98,178 & $85,096 \pm 4226$ & $3,148,536$ \\
Clean reads & 65,283 & 95,316 & $80,134 \pm 4088$ & $2,964,954$ \\
Q20 values & 79.06 & 88.77 & $84.75 \pm 2.64$ & $/$ \\
Efficiencies & 90.70 & 97.95 & $94.19 \pm 2.08$ & $/$ \\
\hline
\end{tabular}

SD indicates standard deviation. Diagonal indicates no count

sample with the fewest sequences. Subsequent analyses were performed based on the normalized data.

Alpha diversity was used to describe community richness and diversity for each sample through four indices (Chao1, ACE, Shannon, and Simpson). All the indices were calculated with QIIME v1.7.0 (Caporaso et al. 2010). Variations in these indices among the different parrot species were assessed using ANOVAs in IBM SPSS statistics 19 (Field 2005). Differences in microbial communities were measured using weighted UniFrac distances, which indicate beta diversity, and were calculated using QIIME v1.7.0 (Caporaso et al. 2010). The statistical significance of the differences was estimated by Anosim in $\mathrm{R}$ v2.15.3 (Beck et al. 2011). To visualize the differences, UPGMA trees based on weighted UniFrac distance were generated in QIIME v1.7.0 (Caporaso et al. 2010). Linear discriminant analysis effect size (LEfSe) was performed using the network tool developed by Segata et al. (2011) to find the key OTUs causing differences. The relationships between Lactobacillus and the gut microbial compositions of parrots were estimated using Pearson correlation analysis in SPSS statistics 19 (Field 2005).

\section{Results}

\subsection{Sequencing and metadata}

We obtained 3,148,536 reads from the hyper-variable V3-V4 region of the 16S rRNA gene from the 37 fecal samples (Table 1). A total of 2,964,954 clean reads were identified after deleting the chimeric reads (Table 1). The average efficiency of sequencing was $94.19 \pm 2.08 \%$ (average \pm standard deviation), ranging from $90.7 \%$ to $97.95 \%$ (Table 1). The values of Q20 for each sample ranged from $79.06 \%$ to $88.77 \%$, and the average was $84.75 \pm 2.64 \%$ (Table 1). All clean reads were clustered into 2909 OTUs with $\geq 97 \%$ sequence similarity. The number of OTUs in each sample ranged from 109 to 722 , and the average was $353 \pm 160$.

\subsection{Core gut microbiomes}

There were 1441 species-specific OTUs and 105 OTUs common in the gut microbiomes of the nine species of parrots (Fig. 1). Although common OTUs accounted for only $3.61 \%$ of total OTUs, they were dominant in the gut microbiomes (Figs. 1 \& 2). To be more specific, the Firmicutes (55.45 \pm $33.85 \%)$, Proteobacteria $(27.03 \pm 25.35 \%)$, Actinobacteria $(10.68 \pm 19.48 \%)$, Bacteroidetes $(4.58 \pm 5.71 \%)$, and Cyanobacteria $(1.17 \pm 3.16 \%)$ were the dominant phyla and were detected in all fecal samples (Fig. 2a). At the genus level, Lactobacillus (31.05 $\pm 35.84 \%)$, Ralstonia $(12.11 \pm 18.49 \%)$, Clostridium sensu stricto $1(8.76 \pm 17.77 \%)$, Candidatus Arthromitus (5.08 $\pm 14.86 \%)$, Acinetobacter (3.82 $\pm 7.36 \%)$, Kocuria (3.75 $\pm 12.69 \%)$, Escherichia-Shigella (3.29 \pm $3.98 \%)$, Planococcus $(3.21 \pm 13.65 \%)$, Rhodococcus ( $2.48 \pm$ $5.97 \%)$, and Staphylococcus $(2.45 \pm 3.35 \%)$ were the
Fig. 1 Flower diagram showing the common OTUs in the gut microbiomes of the nine parrot species

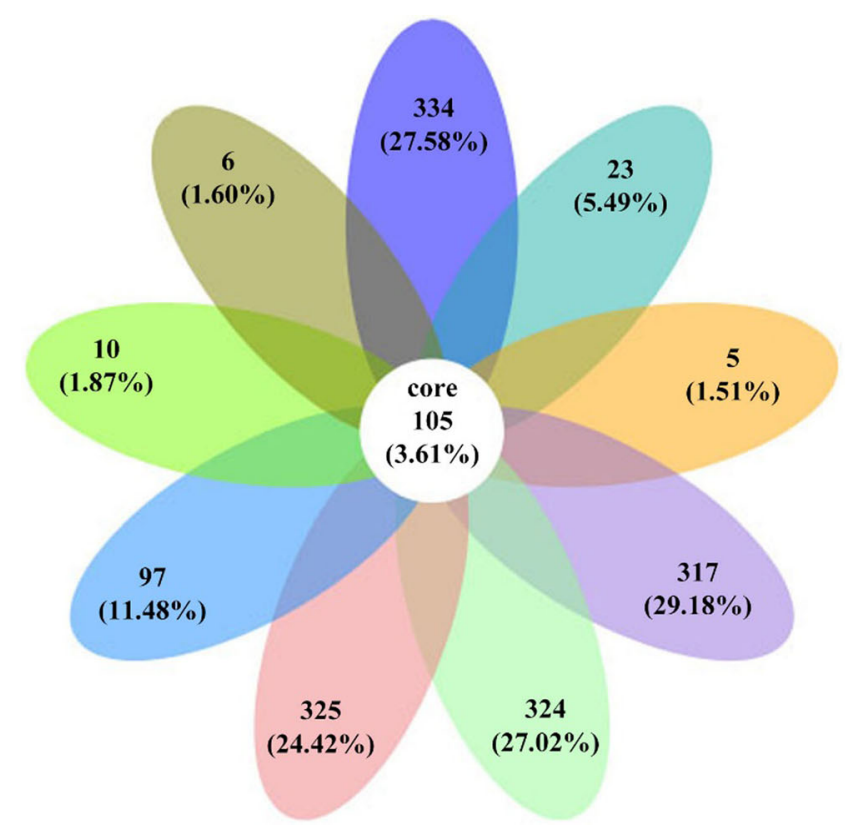


Fig. 2 Average relative abundance of OTUs at the phylum (a) and genus (b) level in the gut microbiomes of the nine parrot species
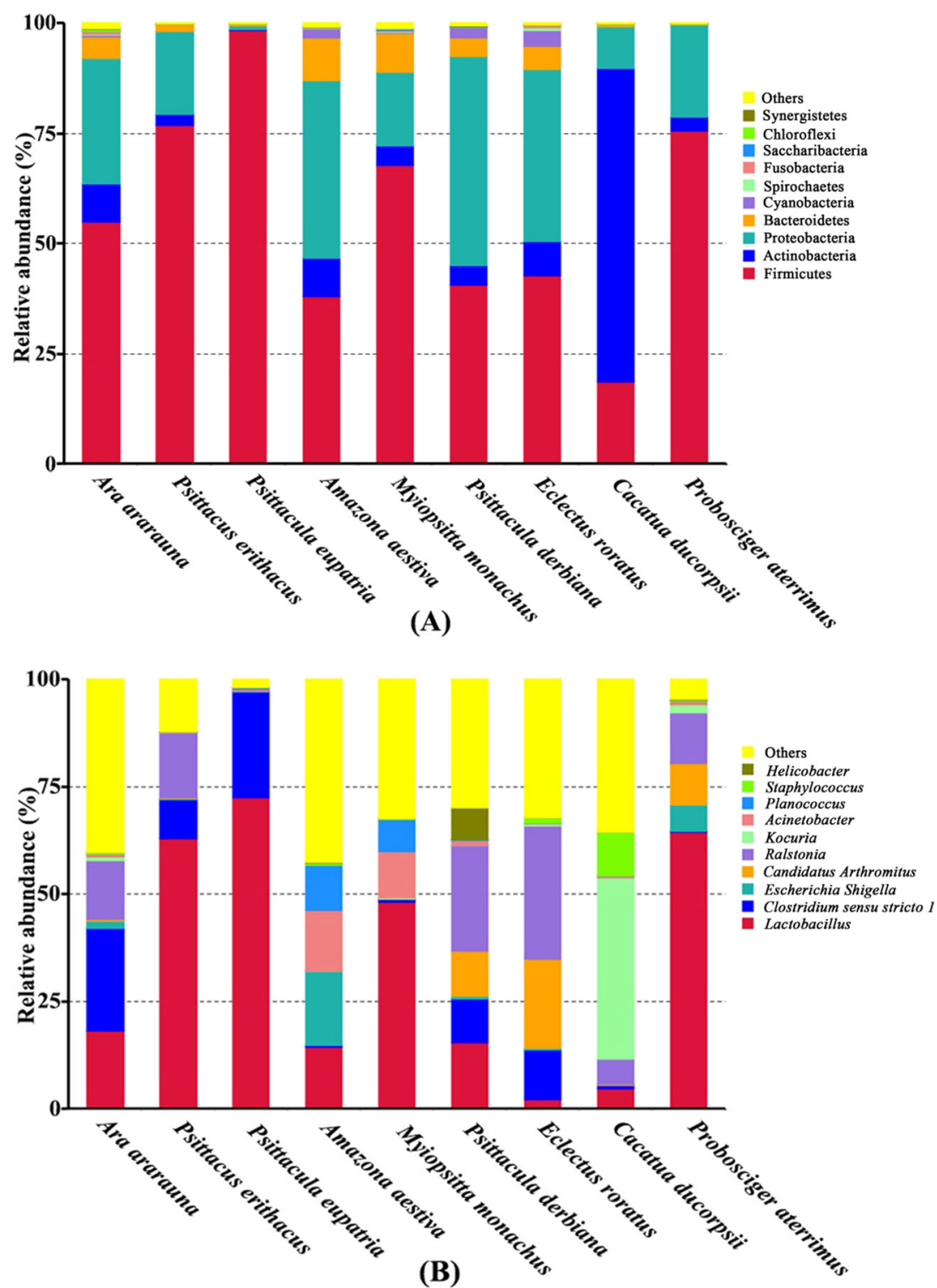

(B)

dominant components of the gut microbiomes in these parrots (Fig. 2b). Although there were dominant bacteria in the guts of these parrots, the relative abundance of dominant bacteria varied among parrot species (Fig. 2).

\subsection{Differences in gut microbiomes among parrot species}

The average Chao 1, Ace, Shannon, and Simpson values for all nine species of parrots were $386.281 \pm 134.974,389.982 \pm$ $118.395,3.630 \pm 0.939$, and $0.736 \pm 0.113$, respectively (Fig. 3). The richness of gut microbiomes differed sharply among parrot species. The Chaol and Ace values for $P$. erithacus, $P$. eupatria, and $P$. aterrimus were significantly lower than those of the other six species $(\mathrm{P}<0.05$, Fig. 3). While the Shannon and Simpson values varied with parrot species, these differences were not statistically significant

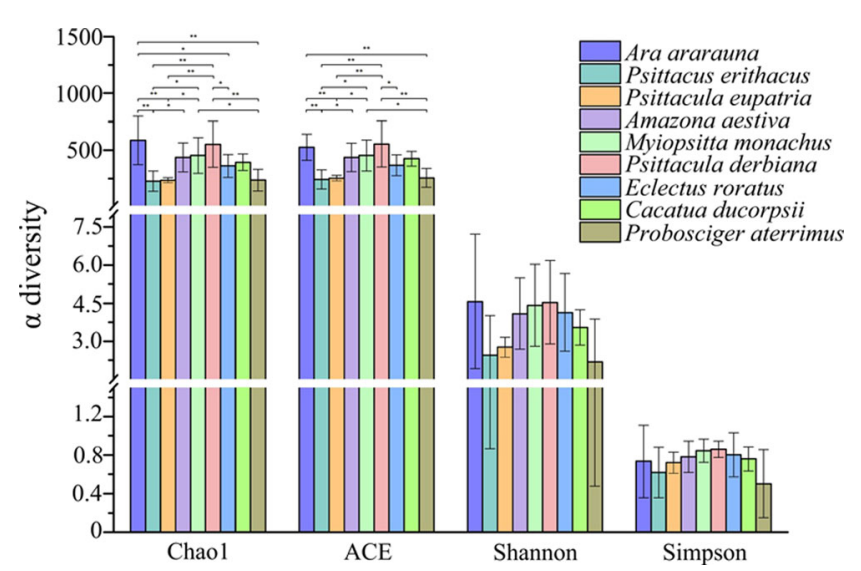

Fig. 3 Comparisons of the richness and diversity of gut microbiomes among nine parrot species. Significance differences are indicated as “**” $(P<0.01)$, “*” $(\mathrm{P}<0.05)$ 
Table 2 Differences in gut microbial compositions of nine parrot species

\begin{tabular}{|c|c|c|c|c|c|c|c|c|c|}
\hline & A. ararauna & P. erithacus & P. eupatria & A. aestiva & M. monachus & P. derbiana & E. roratus & C. ducorpsii & P. aterrimus \\
\hline A. ararauna & & - & - & - & - & - & - & - & - \\
\hline P. erithacus & 0.125 & & - & - & - & - & - & - & - \\
\hline P. eupatria & 0.352 & 0.037 & & - & - & - & + & - & - \\
\hline A. aestiva & 0.100 & 0.275 & 0.518 & & - & - & + & + & - \\
\hline M. monachus & 0.269 & 0.075 & 0.159 & 0.080 & & + & + & + & - \\
\hline P. derbiana & -0.138 & 0.175 & 0.415 & 0.152 & 0.364 & & - & - & - \\
\hline E. roratus & 0.000 & 0.306 & 0.569 & 0.268 & 0.588 & -0.168 & & + & - \\
\hline C. ducorpsii & 0.519 & 0.333 & 1.000 & 0.446 & 0.713 & 0.456 & 0.559 & & - \\
\hline P. aterrimus & 0.037 & -0.074 & 0.333 & 0.180 & 0.026 & 0.169 & 0.333 & 0.630 & \\
\hline
\end{tabular}

$\mathrm{R}$ values are below the diagonal, the significance of their differences is above. When $\mathrm{R}>0$, the difference between species is greater. When $\mathrm{R}<0$, the difference within species is greater. Significance of difference is indicated as “+” $(P<0.05)$ and "-” $(P>0.05)$

(P>0.05, Fig. 3). In addition, there was no evidence of similarity in the richness and diversity of the gut microbiomes of closely related parrots (Fig. 3).

Gut microbial compositions were similar within species, based on a global $\mathrm{R}$ value of $0.254(P=0.04)$, and almost all $\mathrm{R}$ values were greater than zero (Table 2 ). However, differences in gut microbial composition among species did not reflect host phylogenies, as the $\mathrm{R}$ values between some closely related parrots were greater than those between distantly related parrots (Table 2). For instance, the $\mathrm{R}$ value between $P$. eupatria and $P$. derbiana in the same genus was not minimum (Table 2). To visualize the differences, UPGMA trees based on weighted
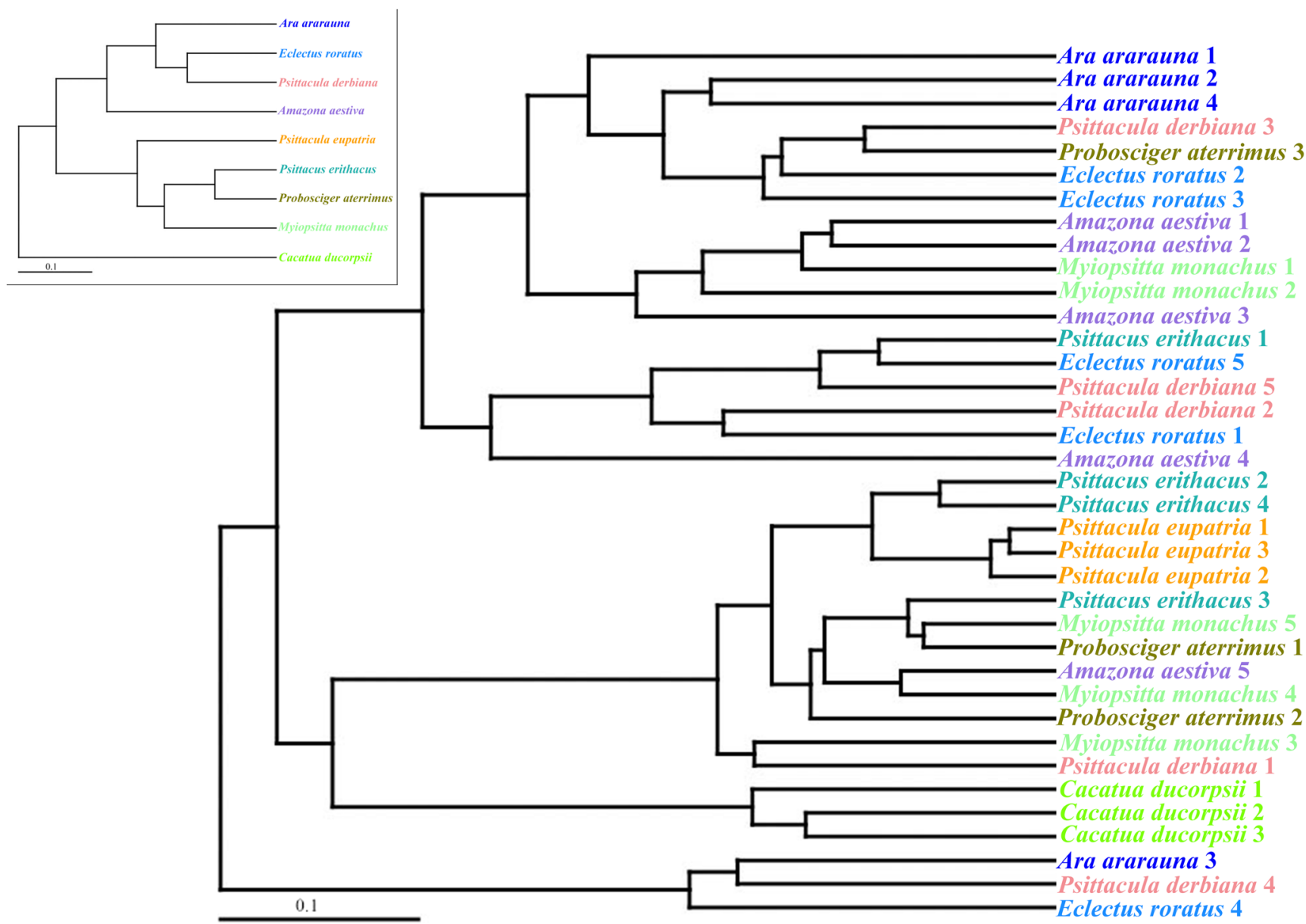

Fig. 4 UPGMA trees of gut microbiomes of the nine parrot species based on weighted UniFrac distance. The main and inset trees show the phylogenies at the individual and species levels, respectively 
C: Unidentified Acidobacteria P: Actinobacteria

O: Micrococcales G: Kocuria

S: Kocuria carniphila

F: Micrococcaceae

F: Brevibacteriaceae

S: Brevibacterium epidermidis

G: Staphylococcus

G: Brevibacterium

F: Dermabacteraceae

F: Staphylococcaceae

G: Brachybacterium

S: Brachybacterium paraconglomeratum

S: Staphylococcus equorum

F: Dermatophilaceae

C: Betaproteobacteria

O: Burkholderiales

F: Burkholderiaceae G: Ralstonia

F: Clostridiaceae 1

G: Candidatus Arthromitus

S: Ralstonia insidiosa

P: Cyanobacteria

C: Chloroplast

G: Unidentified Chloroplast O: Unidentified Chloroplast

F: Unidentified Chloroplast

C: Alphaproteobacteria

O: Campylobacterales

C: Epsilonproteobacteria

F: Helicobacteraceae

G: Helicobacter

F: Oxalobacteraceae

G: Massilia

S: Lactobacillus reuteri

C: Gammaproteobacteria

O: Enterobacteriales

F: Enterobacteriaceae

G: Escherichia Shigella

O: Pseudomonadales

F: Moraxellaceae

S: Escherichia coli

G: Acinetobacter

S: Acinetobacter johnsonii

O: Bacillales

F: Planococcaceae

S: Planococcus salinarum

G: Planococcus

P: Bacteroidetes

O: Corynebacteriales

F: Nocardiaceae

G: Rhodococcus

S: Rhodococcus erythropolis

O: Vibrionales

F: Vibrionaceae

O: Flavobacteriales

C: Flavobacteriia

F: Flavobacteriaceae

G: Photobacterium

F: Carnobacteriaceae

G: Alkalibacterium

G: Flavobacterium

O: Sphingobacteriales

C: Sphingobacteriia

F: Sphingobacteriaceae

P: Firmicutes

F: Lactobacillaceae

O: Lactobacillales

G: Lactobacillus

C: Bacilli

S: Lactobacillus salivarius

G: Clostridium sensu stricto 1

S: Clostridium butyricum

S: Lactobacillus johnsonii

S: Lactobacillus murinus

LDA SCORE $(\log 10)$

Cacatua ducorpsii Eclectus roratus

Psittacula derbiana

Myiopsitta monachus

Amazona aestiva

Psittacula eupatria

Psittacus erithacus 
Fig. 5 The key OTUs causing differences in gut microbial composition of the nine parrot species. These OTUs had the highest differences in abundance among the nine parrot species, but they were not unique to some parrots. Phylum, class, order, family, genus, and species are indicated by $\mathrm{P}, \mathrm{C}, \mathrm{O}, \mathrm{F}, \mathrm{G}$, and $\mathrm{S}$, respectively

UniFrac distance were constructed. Gut microbiomes did not cluster according to host species (Fig. 4). Except for C. ducorpsii and P. eupatria, the gut microbiomes of individual parrots mixed desultorily (Fig. 4). At the species level, the clustering of gut microbiomes was also inconsistent with host phylogenies. For example, the gut microbiomes of $P$. eupatria and $P$. derbiana were separated by the gut microbiome of P. aterrimus, which belong to Cacatuinae (Fig. 4).

LEfSe was performed to find the key OTUs causing the differences in gut microbial composition among the nine species of parrots. The relatively high abundance of 76 OTUs in specific hosts caused the observed differences (Fig. 5). These OTUs were mainly distributed in A. aestiva, C. ducorpsii, and $E$. roratus, while not one was found in $A$. ararauna and P. aterrimus (Fig. 5). The key OTUs at the genus level were Kocuria, Staphylococcus, Brevibacterium, Brachybacterium, Ralstonia, Candidatus Arthromitus, Unidentified Chloroplast, Helicobacter, Massilia, Escherichia Shigella, Acinetobacter, Planococcus, Rhodococcus, Photobacterium, Alkalibacterium, Flavobacterium, Lactobacillus, and Clostridium sensu stricto 1 (Fig. 5).

\subsection{Effects of Lactobacillus on gut microbial composition of parrots}

Considering the high abundance of Lactobacillus and its variability among parrot species, the effects of these bacteria on gut microbial composition were estimated. The $\alpha$ diversity values decreased sharply with increases in the relative abundance of Lactobacillus, indicating that the relative abundance of Lactobacillus was negatively correlated with the richness and diversity of gut microbiomes $(-0.619 \leq \mathrm{r} \leq-0.489$, Fig. 6). The relative abundance of Lactobacillus was also inversely related with eight other bacterial genera, especially Ralstonia and Rhodococcus $(-0.6<\mathrm{r}<-0.2$, Fig. 7). Overall, Lactobacillus might alter the gut microbial composition of parrots.

\section{Discussion}

\subsection{Common gut microbiomes shared by parrots and other herbivorous birds}

We observed a core gut microbiome common to all nine species of parrots (Figs. $1 \& 2$ ). The core gut microbiome at the phylum level was mainly composed of the Firmicutes, Proteobacteria, and Actinobacteria (Fig. 2). These bacterial phyla are also dominant in the guts of kakapo and other herbivorous birds (Waite et al. 2012; Waite et al. 2014; Yang et al. 2016; Zhao et al. 2017). However, the dominant bacterial genera can vary among bird species (Waite et al. 2014; Yang et al. 2016; Zhao et al. 2017). Even in the same host species, there were often changes in the relative abundance of the common bacterial genera. There are obvious differences in the bacterial genera of the gut microbiome of juvenile and adult kakapo in different populations (Waite et al. 2014). Even so, Clostridium and Lactobacillus dominate the gut microbiomes of most herbivorous birds, as shown here and in previous studies (Waite et al. 2014; Yang et al. 2016; Zhao et al. 2017).

The compositions of gut microbiomes in herbivorous birds might be related to their diets. For instance, the high relative abundance of Clostridium may reflect the ability of this genus to effectively catabolize the high-fiber diets of herbivorous avian hosts (Zhao et al. 2017). In addition, the relative abundance of Lactobacillus in the nine species of parrots was much higher than previous studies, which might be related to the commercial feed used in this zoo, which contained Lactobacillus.

\subsection{Gut microbiomes of parrots shaped by both host and environmental factors}

Although the parrots were away from their natural habitats and living in a zoo, the gut microbial compositions individuals of the same species were more similar to each other than to those of other species (Global $\mathrm{R}=0.254, P=0.04$, Table 2). The differences in gut microbiomes among parrot species might have been caused by 76 OTUs (Fig. 5). These OTUs might be related to differences in food preference and physiological activities. The parrots could choose food because abundant commercial feed, fruits, and vegetables were provided daily. Many Chloroplast were found in the fecal samples of

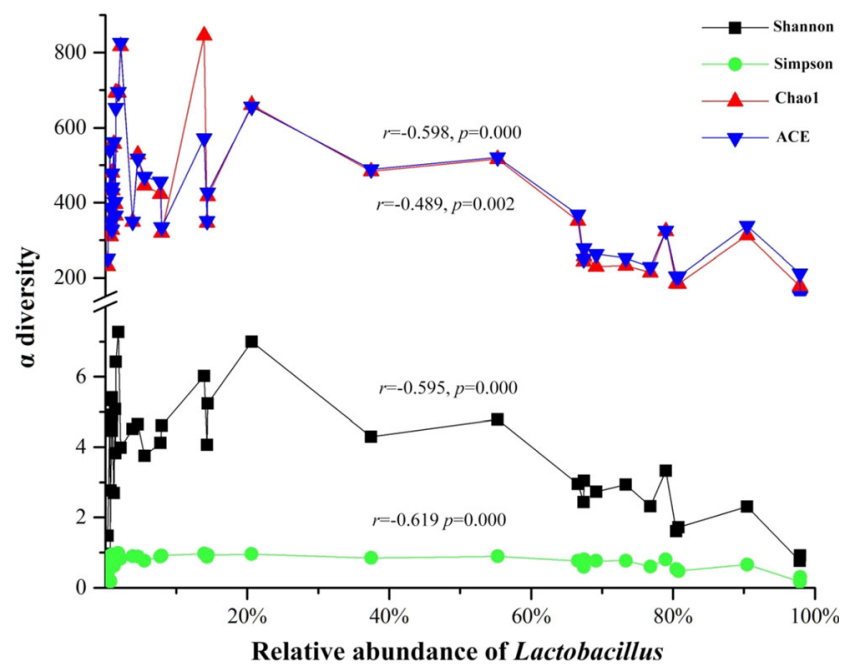

Fig. 6 Line chart showing the relationships between the relative abundance of Lactobacillus and $\alpha$ diversity 
E. roratus, which might indicate these parrots preferred fruits and vegetables. However, plenty of Chloroplast also might be caused by poor digestive capacity. Abundant Lactobacillus were found in the fecal samples of M. monachus, P. erithacus, and P. eupatria, which might indicate that these parrots ate more commercial feed. Some bacteria might interact with hosts and might be transmitted from generation to generation (Uenishi et al. 2007; Brooks et al. 2017; Kohl et al. 2018).

In addition to stable bacteria, transient bacteria can inhabit host guts through stochastic processes associated with the environment (Brooks et al. 2017; Kohl et al. 2018). In our study, we observed three negative $\mathrm{R}$ values in Anosim (Table 2), which may be related to transient bacteria. The UPGMA trees of gut microbiomes at individual and species levels were not in accord with host phylogenies (Fig. 4), also suggesting the presence of transient bacteria. Similar diets and host genetics might lead to intricate relationships in the gut microbiomes of the nine species of parrots (Nelson et al. 2013). Taken together, gut microbiomes of parrots appear to have been shaped by both host and environmental factors.

\subsection{Potential effects of Lactobacillus on the gut microbiomes of parrots}

Lactobacillus might benefit hosts by protecting the hosts against potential invasions by pernicious bacteria and promoting the absorption of protein, monosaccharides, calcium and magnesium (Hemarajata and Versalovic 2013; Valeriano et al. 2017). Lactobacillus has become the most common probiotic in commercial feed (Bai et al. 2013; Phuoc and Jamikorn 2017). Adding Lactobacillus into basic animal diets has

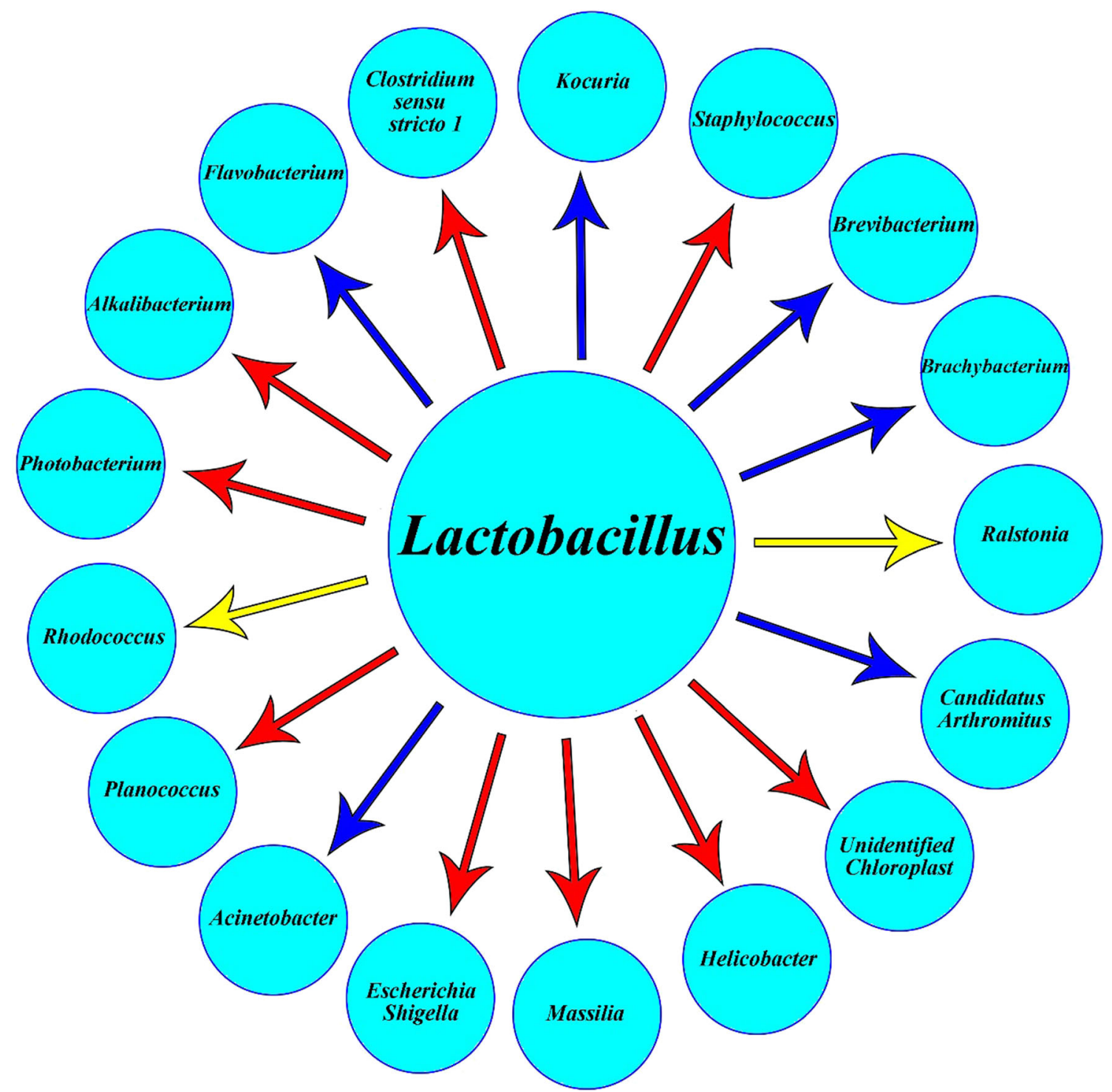

Fig. 7 The relationships between the relative abundance of Lactobacillus and other bacteria. Red, blue and yellow arrows indicate little to no correlations $(0<|\mathrm{r}|<0.2)$, weak negative correlations $(0.2<|\mathrm{r}|<0.4)$ and moderate negative correlations $(0.4<|\mathrm{r}|<0.6)$, respectively 
become popular (De Angelis et al. 2006). A commercial feed with Lactobacillus was used in the zoo, but we did not see evidence that it could effectively withhold maleficent bacteria (such as Staphylococcus, Helicobacter, and Escherichia Shigella) in parrots (Figs. $6 \&$ 7). Indeed, we found that Lactobacillus might significantly lessen the richness and diversity of gut microbiomes in parrots (Figs. $6 \& 7$ ); however, we did not characterize the effects on physiological activities of hosts. Therefore, more research is needed to clarify the influence of Lactobacillus on the gut microbiomes of parrots, which will offer guidance for adjusting parrot diets.

\subsection{Limitations of this study}

Parrots are divided into three families, and a total of around 400 species of parrots exist in the world (IUCN 2018). Subjects for this study only included nine species from two families. Moreover, all the parrots were kept in the same zoo. This study may not exactly reflect the gut microbiomes of all parrots in natural habitats. Many host factors (such as gender, actual age, and health condition) and environmental factors (such as diet composition and microbiology in the environment) can influence the gut microbiomes of parrots. In a study on the influence of bird age and hand rearing on the fecal microbiome of the kakapo, the microbial community structure of juvenile and adult kakapos changed over time, and the abundance of key OTUs was related to antibiotic treatment and captivity (Waite et al. 2014).

Understanding the differences in gut microbiomes among parrots living in different conditions would contribute to finding the key bacteria that affect parrots' physiological activities. However, the dynamic changes in gut microbiomes were not studied here. In addition, the study of gut microbiomes is susceptible to environmental contamination, which can be mitigated using controls, but no controls were used in this study. Improved experimental protocols would lead to more impactful studies of gut microbiomes to better conserve parrots.

Acknowledgements We thank Rong Chen, Zhao-Shui Fu and GuoDong Wang from the Nanjing Hongshan Forest Zoo for providing fecal samples. This work was supported by the National Natural Science Foundation of China (No. 31800453), the Natural Science Foundation of Jiangsu Province of China (No. BK20160927), the Priority Academic Program Development of Jiangsu Higher Education Institutions (PAPD), the Innovation and Entrepreneurship Training Program for College Students of China (201710298043Z), and a grant from the Science and Technology Department of Sichuan Province of China (2017TJPT0031).

Open Access This article is distributed under the terms of the Creative Commons Attribution 4.0 International License (http:// creativecommons.org/licenses/by/4.0/), which permits unrestricted use, distribution, and reproduction in any medium, provided you give appropriate credit to the original author(s) and the source, provide a link to the Creative Commons license, and indicate if changes were made.

\section{References}

Andoh A (2016) Physiological role of gut microbiota for maintaining human health. Digestion 93:176-181

Bai SP, Wu AM, Ding XM, Lei Y, Bai J, Zhang KY, Chio JS (2013) Effects of probiotic-supplemented diets on growth performance and intestinal immune characteristics of broiler chickens. Poult Sci 92:663-670

Beck D, Settles M, Foster JA (2011) OTUbase: an R infrastructure package for operational taxonomic unit data. Bioinformatics 27:17001701

Berg ML, Bennett ATD (2010) The evolution of plumage colouration in parrots: a review. Emu 110:10-20

Bradbury JW, Balsby TJS (2016) The functions of vocal learning in parrots. Behav Ecol Sociobiol 70:293-312

Brooks AW, Kohl KD, Brucker RM, van Opstal EJ, Bordenstein SR (2017) Phylosymbiosis: relationships and functional effects of microbial communities across host evolutionary history. PLoS Biol 15:e1002587

Bush ER, Baker SE, Macdonald DW (2014) Global trade in exotic pets 2006-2012. Conserv Biol 28:663-676

Caporaso JG, Kuczynski J, Stombaugh J, Bittinger K, Bushman FD, Costello EK, Fierer N, Peña AG, Goodrich JK, Gordon JI, Huttley GA, Kelley ST, Knights D, Koenig JE, Ley RE, Lozupone CA, McDonald D, Muegge BD, Pirrung M, Reeder J, Sevinsky JR, Turnbaugh PJ, Walters WA, Widmann J, Yatsunenko T, Zaneveld J, Knight R (2010) QIIME allows analysis of high-throughput community sequencing data. Nat Methods 7:335-336

De Angelis M, Siragusa S, Berloco M, Caputo L, Settanni L, Alfonsi G, Amerio M, Grandi A, Ragni A, Gobbetti M (2006) Selection of potential probiotic lactobacilli from pig feces to be used as additives in pelleted feeding. Res Microbiol 157:792-801

Edgar RC (2013) UPARSE: highly accurate OTU sequences from microbial amplicon reads. Nat Methods 10:996-998

Edgar RC, Haas BJ, Clemente JC, Quince C, Knight R (2011) UCHIME improves sensitivity and speed of chimera detection. Bioinformatics 27:2194-2200

Field A (2005) Discovering statistics using SPSS. Sage Press, London

Haas BJ, Gevers D, Earl AM, Feldgarden M, Ward DV, Giannoukos G, Ciulla D, Tabbaa D, Highlander SK, Sodergren E, Methé B, TZ DS, Consortium HM, Petrosino JF, Knight R, Birren BW (2011) Chimeric 16S rRNA sequence formation and detection in sanger and 454-pyrosequenced PCR amplicons. Genome Res 21:494-504

Heinsohn R, Buchanan KL, Joseph L (2018) Parrots move to Centre stage in conservation and evolution. EMU 118:1-6

Hemarajata P, Versalovic J (2013) Effects of probiotics on gut microbiota: mechanisms of intestinal immunomodulation and neuromodulation. Ther Adv Gastroenterol 6:39-51

IUCN (2018) The IUCN red list of threatened species. http://www. iucnredlist.org

Kers JG, Velkers FC, Fischer EAJ, Hermes GDA, Stegeman JA, Smidt H (2018) Host and environmental factors affecting the intestinal microbiota in chickens. Front Microbiol 9:235

Kohl KD, Dearing MD, Bordenstein SR (2018) Microbial communities exhibit host species distinguishability and phylosymbiosis along the length of the gastrointestinal tract. Mol Ecol 27:1874-1883

Martin M (2011) Cutadapt removes adapter sequences from highthroughput sequencing reads. EMBnet J 17:10-12

Nelson TM, Rogers TL, Carlini AR, Brown MV (2013) Diet and phylogeny shape the gut microbiota of Antarctic seals: a comparison of wild and captive animals. Environ Microbiol 15:1132-1145

Olah G, Butchart SHM, Symes A, Medina Guzmán I, Cunningham R, Brightsmith DJ, Heinsohn R (2016) Ecological and socio-economic factors affecting extinction risk in parrots. Biodivers Conserv 25: 205-223 
Phuoc TL, Jamikorn U (2017) Effects of probiotic supplement (Bacillus subtilis and Lactobacillus acidophilus) on feed efficiency, growth performance, and microbial population of weaning rabbits. Asian Australas J Anim Sci 30:198-205

Quast C, Pruesse E, Yilmaz P, Gerken J, Schweer T, Yarza P, Peplies J, Glöckner FO (2013) The SILVA ribosomal RNA gene database project: improved data processing and web-based tools. Nucleic Acids Res 41:D590-D596

Rodríguez-López R (2016) Environmental enrichment for parrot species: are we squawking up the wrong tree? Appl Anim Behav Sci 180:1-10

Rothschild D, Weissbrod O, Barkan E, Kurilshikov A, Korem T, Zeevi D, Costea PI, Godneva A, Kalka IN, Bar N, Shilo S, Lador D, Vila AV, Zmora N, Pevsner-Fischer M, Israeli D, Kosower N, Malka G, Wolf BC, Avnit-Sagi T, Lotan-Pompan M, Weinberger A, Halpern Z, Carmi S, Fu J, Wijmenga C, Zhernakova A, Elinav E, Segal E (2018) Environment dominates over host genetics in shaping human gut microbiota. Nature 555:210-215

Sanders JG, Powell S, Kronauer DJ, Vasconcelos HL, Frederickson ME, Pierce NE (2014) Stability and phylogenetic correlation in gut microbiota: lessons from ants and apes. Mol Ecol 23:1268-1283

Schloss PD, Westcott SL, Ryabin T, Hall JR, Hartmann M, Hollister EB, Lesniewski RA, Oakley BB, Parks DH, Robinson CJ, Sahl JW, Stres B, Thallinger GG, Van Horn DJ, Weber CF (2009) Introducing mothur: open-source, platform-independent, community-supported software for describing and comparing microbial communities. Appl Environ Microbiol 75:7537-7541

Segata N, Izard J, Waldron L, Gevers D, Miropolsky L, Garrett WS, Huttenhower C (2011) Metagenomic biomarker discovery and explanation. Genome Biol 12:R60
Sekercioglu CH (2006) Increasing awareness of avian ecological function. Trends Ecol Evol 21:464-471

Simberloff D (2001) Threatened birds of the world. Auk 118:1112-1113

Uenishi G, Fujita S, Ohashi G, Kato A, Yamauchi S, Matsuzawa T, Ushida K (2007) Molecular analyses of the intestinal microbiota of chimpanzees in the wild and in captivity. Am J Primatol 69:367-376

Valeriano VD, Balolong MP, Kang DK (2017) Probiotic roles of Lactobacillus sp. in swine: insights from gut microbiota. J Appl Microbiol 122:554-567

Waite DW, Deines P, Taylor MW (2012) Gut microbiome of the critically endangered New Zealand parrot, the kakapo (Strigops habroptilus). PLoS One 7:e35803

Waite DW, Eason DK, Taylor MW (2014) Influence of hand rearing and bird age on the fecal microbiota of the critically endangered kakapo. Appl Environ Microbiol 80:4650-4658

Wang M, Monaco MH, Donovan SM (2016) Impact of early gut microbiota on immune and metabolic development and function. Semin Fetal Neonatal Med 21:380-387

Xu LQ, Liu XD, Wu LB, Sun LG, Zhao JJ, Chen L (2016) Decline of recent seabirds inferred from a composite 1000-year record of population dynamics. Sci Rep 6:35191

Yang Y, Deng Y, Cao L (2016) Characterising the interspecific variations and convergence of gut microbiota in Anseriformes herbivores at wintering areas. Sci Rep 6:32655

Zhao G, Zhou L, Dong Y, Cheng Y, Song Y (2017) The gut microbiome of hooded cranes (Grus monacha) wintering at Shengjin Lake, China. Microbiologyopen 6:e447

Publisher's note Springer Nature remains neutral with regard to jurisdictional claims in published maps and institutional affiliations. 